\title{
Peak flow diaries in childhood asthma are unreliable
}

A W A Kamps, R J Roorda, P L P Brand

\begin{abstract}
Background-A study was undertaken to investigate the compliance with and accuracy of peak flow diaries in childhood asthma.

Methods-Forty asthmatic children (5-16 years) were asked to perform peak flow measurements twice daily for 4 weeks by means of an electronic peak flow meter and to record values in a written diary. Patients and parents were unaware that the device stored the peak flow values on a microchip. In random order, half of the patients were only told that the device allowed for more accurate assessment of peak flow while the other half were told that accurate recording of peak flow was important because the results would be used in guiding adjustments to treatment. Data in the written diary (reported data) were compared with those from the electronic diary (actual data).
\end{abstract}

Results-In the entire study population the mean (SD) actual compliance (77.1 $(20.5) \%)$ was much lower than the mean reported compliance (95.7 (9.1)\%) $(95 \%$ CI for difference $12.7 \%$ to $24.4 \%$ ) The percentage of correct peak flow entries decreased from $56 \%$ to $<50 \%$ from the first to the last study week $(p<0.04)$, mainly as a result of an increase in self-invented peak flow entries. Results were comparable for both groups. For incorrect peak flow entries the mean difference between written and electronically recorded entries ranged from -72 to $341 / \mathrm{min}$ per patient. Conclusions-Peak flow diaries kept by asthmatic children are unreliable. Electronic peak flow meters should be used if peak flow monitoring is required in children with asthma.

(Thorax 2001;56:180-182)

Paediatrics, Division

of Paediatric

Pulmonology, Isala

Klinieken,

Weezenlanden

Hospital, PO Box

10500, 8000 GM Zwolle,

The Netherlands

A W A Kamps

R J Roorda

P L P Brand

Correspondence to: Dr P L P Brand

P.L.P.Brand@isala.nl

Received 14 September 2000

Returned to authors

21 October 2000

Revised version received

6 November 2000

Accepted for publication

24 November 2000

Keywords: asthma; children; home monitoring; peak expiratory flow; compliance

Current guidelines for management of childhood asthma advocate the use of long term home monitoring of peak flow to provide an "objective measurement for the course of the disease and the patient's response to therapy" ${ }^{12}$ In self-management plans for children with asthma, adjustments to therapy are usually based on peak flow values recorded in a diary. ${ }^{34}$ Although peak flow diaries thus play a prominent role in the management of this very common disorder, limited published evidence is available on the reliability of peak flow diaries kept by asthmatic children and their parents.
Adults have been shown to be unreliable in keeping peak flow diaries for themselves, ${ }^{5-8}$ but parents may be more compliant to doctor's orders for their children's illnesses than for their own. In one study in children with asthma, the number of correct peak flow entries decreased from $80 \%$ during the first week to $50 \%$ during the third week of peak flow recording. ${ }^{9}$ This study, however, was performed in African-American and Hispanic underprivileged children from an inner city environment visiting the emergency room for acute asthma whose compliance with treatment was known to be poor. ${ }^{10}$

We now report the results of a study examining the accuracy and reliability of peak flow diaries in white asthmatic children with relatively stable asthma.

\section{Methods}

PATIENTS

Forty consecutive asthmatic children aged 6-15 years of white ethnic origin who attended our paediatric outpatient asthma clinic for a regular follow up visit agreed to participate in the study. None lived in underprivileged low socioeconomic conditions and all had been under our care for at least one year before enrollment in the study. All patients and parents had received our usual comprehensive education programme on the causes of asthma, on measures to reduce allergen and passive smoke exposure, and on inhaled drug therapy. All children had moderately severe persistent asthma and were clinically stable on maintenance therapy with inhaled corticosteroids. They appeared to be well motivated to comply with treatment. Most patients had used a peak flow meter previously, but none had used a peak flow meter on a regular basis in the 6 months prior to entering the study.

\section{STUDY DESIGN}

Patients were given a portable electronic peak flow meter (Vitalograph 2110, Vitalograph Ltd, Buckingham, UK) and were instructed in the correct use of the device. Before taking the device home patients had to demonstrate a correct manoeuvre. Patients were asked to perform peak flow measurements in the morning between 06.00 and 10.00 hours and in the evening between 18.00 hours and 22.00 hours. They were instructed to perform three peak flow manoeuvres and to record the highest of these three attempts in a written diary for 4 weeks.

Patients and parents were unaware that the electronic peak flow meter automatically stored time on a microchip memory. They were told the peak flow values together with the date and 


\section{Compliance}

Number of recorded peak flow values expressed as a percentage of the number of required peak flow values (twice daily for 4 weeks, $\mathrm{n}=56$ ).

Reported compliance: compliance calculated for the written peak flow diary.

Actual compliance: compliance calculated for the electronic peak flow diary.

\section{Accuracy}

For the following variables percentages of occurrence were computed:

Correct peak expiratory flow: recorded peak flow in the written diary is identical to the highest peak flow value at the appropriate time point in the electronic diary.

Incorrect peak expiratory flow: recorded peak flow in the written diary is not identical to the highest of peak flow values recorded at a given time point.

Self-invented peak expiratory flow: peak flow value is not recorded in the electronic diary but is in the written diary.

Missing peak expiratory flow: peak flow value is recorded neither in the electronic nor in the written diary.

Box 1 Variables computed from each peak flow diary.

that the device was novel in that it could record peak flow digitally and electronically, allowing for more accurate assessment of peak flow. Half of the patients received no other information than this ("basic information", BI group) while the other half of the patients were explicitly told that accurate recording of peak flow was important because peak flow values would be used in guiding adjustments to treatment ("extended information", EI group). Allocation to these two groups was done at random using a random number table generated by a computer statistical package (SPSS).

ANALYSIS OF DATA

At the end of the 4 week study period patients returned to the clinic for a check up. The data

Table 1 Mean (SD) actual compliance for patients of both groups for each week of the study period

\begin{tabular}{llll}
\hline & BI group & EI group & $\begin{array}{l}\text { 95\% CI for difference between } \\
\text { BI and EI group }\end{array}$ \\
\hline Week 1 & $82.1(17.9)$ & $88.0(13.3)$ & -16.0 to 4.1 \\
Week 2 & $74.6(24.7)$ & $84.7(18.4)$ & -24.0 to 3.9 \\
Week 3 & $72.1(28.6)$ & $76.1(30.2)$ & -22.8 to 14.9 \\
Week 4 & $63.0(32.6)$ & $73.7(26.7)$ & -30.1 to 8.7 \\
& $\mathrm{p}=0.006 \dagger$ & $\mathrm{p}=0.03 \dagger$ & \\
\hline
\end{tabular}

$\mathrm{BI}=$ basic information; $\mathrm{EI}=$ extended information (see text).

$\star$ Unpaired $t$ test.

†Change over time analysed with repeated measures ANOVA.

Table 2 Mean (SD) percentage of correct, incorrect, missing, and self-invented peak flow entries for both groups

\begin{tabular}{lccl}
\hline & BI group & EI group & $\begin{array}{c}\text { 95\% CI for difference between } \\
\text { BI and EI group }\end{array}$ \\
\hline Correct & $49.1(21.5)$ & $52.2(26.1)$ & -18.4 to 12.3 \\
Incorrect & $22.7(8.6)$ & $28.5(8.6)$ & -16.3 to 4.8 \\
Missing & $5.8(7.6)$ & $6.5(11.6)$ & -7.0 to 5.6 \\
Self-invented & $22.4(23.5)$ & $13.1(12.2)$ & -2.7 to 21.3
\end{tabular}

$\mathrm{BI}=$ basic information; $\mathrm{EI}=$ extended information (see text).

$\star$ Unpaired $t$ test. on the memory chip of each electronic peak flow meter were downloaded into a personal computer. Each electronic peak flow diary was checked for excessive outliers (more than +2 SD from the diary's mean peak flow) that might suggest that an adult or an older sibling had used the device ${ }^{9}$ and such outliers were excluded. Peak flow values recorded outside the required time periods (between 06.00 and 10.00 hours and between 18.00 and 22.00 hours) were also excluded from analysis.

From each diary the variables listed in box 1 were computed. The change in variables over time was analysed using repeated measures ANOVA, comparisons within groups were performed with paired $t$ tests, and comparisons between groups were performed using independent sample $t$ tests. SPSS was used for statistical analyses.

ETHICAL CONSIDERATIONS

The study was approved by the hospital medical ethics review board which agreed that, for the particular purpose of this study, informed consent of patients and parents need not be sought.

\section{Results}

\section{PATIENTS}

All 40 patients (27 boys) completed the study. The mean (SD) age was 9.2 (2.3) years. All patients were using inhaled corticosteroids at a mean (SD) daily dose of 268 (159) $\mu \mathrm{g}$. The mean (SD) forced expiratory volume in one second $\left(\mathrm{FEV}_{1}\right)$ was 103.5 (14.6)\% predicted.

\section{COMPLIANCE}

The mean (SD) reported compliance was 96.6 (6.3) $\%$ in the BI group and $94.8(11.4) \%$ in the EI group $(95 \%$ CI for difference $-4.0 \%$ to $7.6 \%)$. Over the entire study period the mean reported compliance was considerably higher than the mean actual compliance for both the BI group (73.4 (22.4)\%, 95\% CI for difference $12.8 \%$ to $33.7 \%)$ and the EI group (80.9 (18.3)\%, 95\% CI for difference $8.2 \%$ to $19.6 \%)$. There was no significant difference in actual compliance between the two groups (95\% CI for difference $-20.6 \%$ to $5.6 \%$ )

The actual compliance in both groups decreased significantly from week 1 to week 4 (table 1). Overall actual compliance was $<50 \%$ in $12.5 \%$ of patients, $50-75 \%$ in $20 \%$ of patients, and $>75 \%$ in $67.5 \%$ of patients.

\section{ACCURACY}

The mean percentage of correct, incorrect, missing, and self-invented peak flow values are presented in table 2 . The percentage of correct peak flow value entries decreased throughout the study in both groups (table 3). The percentage of incorrect peak flow value entries remained fairly constant throughout the study and was comparable between groups, as was the percentage of missing peak flow values. The percentage of self-invented peak flow entries was slightly higher in the BI group than in the EI group (mean difference 9.3\%; 95\% CI $-2.7 \%$ to $21.3 \%$; table 2 ). In the BI group the percentage of self-invented peak flow entries 
Table 3 Changes over time of the percentage of correct, incorrect, missing, and self-invented peak flow values for both groups

\begin{tabular}{|c|c|c|c|c|c|c|c|c|}
\hline & \multicolumn{4}{|c|}{ BI group } & \multicolumn{4}{|c|}{ EI group } \\
\hline & Week 1 & Week 2 & Week 3 & Week 4 & Week 1 & Week 2 & Week 3 & Week 4 \\
\hline Correct & 56.4 & 49.6 & 49.7 & 39.0 & 56.3 & 58.7 & 48.0 & 48.2 \\
\hline Incorrect & 24.6 & 23.3 & 22.1 & 22.8 & 31.7 & 26.1 & 28.6 & 24.5 \\
\hline Missing & 7.9 & 6.1 & 5.7 & 5.2 & 4.0 & 3.6 & 8.2 & 10.2 \\
\hline Invented & 11.1 & 20.3 & 22.9 & $32.9^{\star}$ & 7.9 & 11.0 & 16.0 & $17.5^{\star}$ \\
\hline
\end{tabular}

* Percentage of self-invented peak flow values increased significantly from week 1 to week 4 in the BI group $(\mathrm{p}=0.001)$ but not in the EI group $(\mathrm{p}=0.28)$.

$\mathrm{BI}=$ basic information; $\mathrm{EI}=$ extended information (see text).

increased almost threefold from week 1 to week $4(\mathrm{p}=0.001)$ while the increase in self-invented peak flow entries in the EI group was not significant $(p=0.28$; table 3$)$.

For incorrect peak flow entries the mean difference between written and electronically recorded peak flow entries ranged from -72 to $34 \mathrm{l} / \mathrm{min}$ per patient. The largest negative and positive differences noted in the entire study population between single electronically recorded peak flow and their corresponding written entries in the dairy were -168 and $163 \mathrm{l} / \mathrm{min}$, respectively.

\section{Discussion}

This study shows that peak flow diaries kept by well informed, well motivated white children with asthma are unreliable. Not unexpectedly, the overall compliance with peak flow monitoring during the 4 week study was considerably higher in this group of affluent white asthmatic children (table 1) than in underprivileged black or Hispanic inner city children studied previously. ${ }^{9}$ The accuracy of the peak flow diaries in the children we studied, however, was surprisingly poor, with up to $25 \%$ of peak flow entries being incorrectly recorded-that is, the patients wrote down a different value in the diary from that actually blown with the peak flow meter. The magnitude of the difference between the actual peak flow value and the one written in the diary could amount to $>160 \mathrm{l} /$ min. In addition, even in this group of well motivated white children, a considerable proportion of peak flow entries were selfinvented - that is, not blown at all but a written entry in the diary anyway. Patients in the BI group recorded such self-invented peak flow values slightly more frequently than patients in the EI group (table 2). This suggests that, if patients know that peak flow values in their diary may lead to adjustment of their current treatment, they are perhaps less likely to invent peak flow values. Nevertheless, even in children who were carefully informed on the importance of monitoring peak flow, only half of the written peak flow values were recorded correctly (table 2). This implies that asthmatic children are not reliable in monitoring and recording peak flow, even for a relatively short period of time.

The implications of these results for clinical practice and research are considerable. In clinical practice peak flow diaries are used very frequently for monitoring childhood asthma and making adjustments to maintenance therapy. ${ }^{12}$ If as many as half of the entries in a peak flow diary are unreliable, then the use of peak flow diaries for asthma self-management is clearly not justified. Patients in our study both overscored and underscored their actual peak flow values in their written diaries. As a result, both undertreatment and overtreatment of childhood asthma is a very real possibility if practitioners base their treatment decisions on the results of written peak flow diaries. Similarly, the results of clinical studies using peak flow diaries as an end point must be seriously questioned if many of the entries in such diaries are either erroneous or self-invented. We propose that, if peak flow monitoring is to be implemented in clinical trials of childhood asthma, only electronic peak flow meters should be used.

This study was performed in a hospital based population of well motivated, well informed white affluent children with relatively stable moderately severe persistent asthma. It is possible that other asthmatic children would be more reliable in keeping a peak flow diary. In particular, children using a peak flow meter in the context of a formal self-management plan with specific therapeutic actions being taken at certain peak expiratory flow levels might produce more reliable diaries. Further studies are therefore needed to evaluate the reliability of peak flow diaries in other populations and settings. If our findings are confirmed in other studies, the recommendation to perform home monitoring of peak flow routinely should be removed from asthma guidelines.

The Vitalograph electronic peak flow meters were kindly provided by Astra Zeneca, The Netherlands.

1 British Thoracic Society, National Asthma Campaign, Royal College of Physicians of London, et al. The British guidelines on asthma management: 1995 review and position statement. Thorax 1997;52(Suppl 1):S1-21

2 National Heart Lung and Blood Institute, National Institutes of Health. International consensus report on diagnosis and treatment of asthma. NHLBI Publication No. 92-3091. Eur Respir F 1992;5:601-41.

3 Madge P, McColl J, Paton J. Impact of a nurse-led home management training programme in children admitted to hospital with acute asthma: a randomised controlled study. Thorax 1997;52:223-8.

4 Charlton I, Antoniou AG, Atkinson J, et al. Asthma at the interface: bridging the gap between general practice and a district general hospital. Arch Dis Child 1994;70:313-8.

5 Verschelden P, Cartier A, L'Archeveque J, et al. Compliance with and accuracy of daily self-assessment of peakwith and accuracy of daily self-assessment of peakmonth period. Eur Respir f 1996;9:880-5.

6 Hyland ME, Kenyon CAP, Allen R, et al. Diary keeping in asthma: comparison of written and electronic methods. BMF 1993;306:487-9.

7 Malo JL, Trudeau C, Ghezzo H, et al. Do subjects investigated for occupational asthma through serial peak expiratory flow measurements falsify their results? F Allergy Clin Immunol 1995;96:601-7.

8 Cote J, Cartier A, Malo JL, et al. Compliance with peak expiratory flow monitoring in home management of asthma. Chest 1998;113:968-72.

9 Redline S, Wright EC, Kattan M, et al. Short-term compliance with peak flow monitoring: results from a study of inner city children with asthma. Pediatr Pulmonol 1996;21: 203-10.

10 Eggleston PA, Malveaux FJ, Butz AM, et al. Medications used by children with asthma living in the inner city. Pediatrics 1998;101:349-54. 